\title{
Activation of transient receptor potential vanilloid 4 increases NMDA-activated current in hippocampal pyramidal neurons
}

\author{
Lin Li $^{1,2}$, Weijun $Q u^{1}$, Libin Zhou ${ }^{1}$, Zihong Lu ${ }^{1}$, Pinghui Jie ${ }^{1}$, Lei Chen ${ }^{1 *}$ and Ling Chen ${ }^{1,2}{ }^{*}$ \\ ${ }^{1}$ Department of Physiology, Nanjing Medical University, Nanjing, China \\ ${ }^{2}$ State Key Laboratory of Reproductive Medicine, Nanjing Medical University, Nanjing, China
}

\section{Edited by:}

Dieter Wicher, Max Planck Institute

for Chemical Ecology, Germany

\section{Reviewed by:}

Christian F. Erxleben, National

Institute of Environmental Health

Sciences, USA

Baruch Minke, Hebrew University of Jerusalem, Israel

Veronica Egger,

Ludwig-Maximilians-Universität,

Germany

\section{*Correspondence:}

Lei Chen and Ling Chen, Department of Physiology, Nanjing Medical

University, No. 140, Hanzhong Road,

Nanjing 210029, China.

e-mail:chenl@njmu.edu.cn;

lingchen@njmu.edu.cn
The glutamate excitotoxicity, mediated through $\mathrm{N}$-methyl-d-aspartate receptors (NMDARs), plays an important role in cerebral ischemia injury. Transient receptor potential vanilloid 4 (TRPV4) can be activated by multiple stimuli that may happen during stroke. The present study evaluated the effect of TRPV4 activation on NMDA-activated current (/NMDA $)$ and that of blocking TRPV4 on brain injury after focal cerebral ischemia in mice. We herein report that activation of TRPV 4 by $4 \alpha-P D D$ and hypotonic stimulation increased / NMDA in hippocampal CA1 pyramidal neurons, which was sensitive to TRPV4 antagonist HC-067047 and NMDAR antagonist AP-5, indicating that TRPV4 activation potentiates NMDAR response. In addition, the increase in /NMDA by hypotonicity was sensitive to the antagonist of NMDAR NR2B subunit, but not of NR2A subunit. Furthermore, antagonists of calcium/calmodulindependent protein kinase II (CaMKII) significantly attenuated hypotonicity-induced increase in /NMDA, while antagonists of protein kinase $C$ or casein kinase II had no such effect, indicating that phosphorylation of NR2B subunit by CaMKII is responsible for TRPV4-potentiated NMDAR response. Finally, we found that intracerebroventricular injection of HC-067047 after 60 min middle cerebral artery occlusion reduced the cerebral infarction with at least a $12 \mathrm{~h}$ efficacious time-window. These findings indicate that activation of TRPV 4 increases NMDAR function, which may facilitate glutamate excitotoxicity. Closing TRPV4 may exert potent neuroprotection against cerebral ischemia injury through many mechanisms at least including the prevention of NMDAR-mediated glutamate excitotoxicity.

Keywords:TRPV4, NMDA receptor, NR2B subunit, phosphorylation, excitotoxicity, cerebral ischemia

\section{INTRODUCTION}

Stroke is a worldwide health problem leading to high rates of death and neurological disability in adults. The mechanisms underlying cerebral ischemia injury are complex, but the intracellular free calcium $\left(\left[\mathrm{Ca}^{2+}\right]_{\mathrm{i}}\right)$ overload has been proved to play a vital role (Szydlowska and Tymianski, 2010). It is generally accepted that during cerebral ischemia, a large amount of glutamate accumulates in the synaptic cleft, which results in excessive calcium influx through $N$-methyl-D-aspartate receptors (NMDARs), to trigger and eventually induce cell death (Paschen, 1996). The glutamate excitotoxicity has long been recognized, however, treatment by directly targeting glutamate receptors has failed in clinical trials either because of intolerable side effects or lack of medical efficacy (Kemp and McKernan, 2002).

Apart from neuronal death, another critically important pathophysiological process in ischemic stroke is the formation of brain edema which includes the cytotoxic and vasogenic edema (Simard et al., 2007). The cytotoxic edema is the initial phase, which is caused by the energy failure leading to intracellular fluid accumulation. With ongoing of ischemia and reperfusion, cytotoxic edema becomes progressively evident and the secondary vasogenic edema eventually develops due to the disruption of blood-brain barrier (BBB). Brain edema is not only an important pathological process during cerebral ischemia, but also contributes to the adverse outcome (Marmarou, 2007; Simard et al., 2007). Therefore, prevention of brain edema or alleviation of brain edema-induced injury is critical for the treatment of cerebral ischemia.

Recently, there is accumulating evidence concerning the involvement of Transient Receptor Potential (TRP) channels, a potentially important calcium influx pathway, in cerebral ischemia injury. For example, TRP melastatin (TRPM) members, especially TRPM2 and TRPM7, are important contributors to the neuronal death following ischemia (Sun et al., 2009; Bai and Lipski, 2010). Besides TRPM channels, other TRP family members might also be responsible for ischemic neuronal injury. Among them, TRP vanilloid 4 (TRPV4) is attracting more and more attention because it can be activated by multiple stimuli including hypotonic stimulation, cell swelling, mild heat $\left(>24-37^{\circ} \mathrm{C}\right)$, arachidonic acid (AA), and its metabolism epoxyeicosatrienoic acids (EETs; Plant and Strotmann, 2007), some of which may happen during stroke. TRPV4 is widely expressed in the central nervous system, including hippocampus, cortex, thalamus, and cerebellum etc. (Kauer and Gibson, 2009). During ischemia, the energy failure results in cytotoxic edema and membrane lipid metabolism disorder causing extra release of free AA (Westerberg et al., 1987; Marmarou, 2007; Simard et al., 2007). Therefore, it is very likely that TRPV4 
is hyper-activated during ischemia, leading to an increase in calcium influx. Blockage of TRPV4 by lowering temperature may be responsible for the neuroprotection against oxygen-glucose deprivation-induced injury (Lipski et al., 2006). TRPV4 blockers $\mathrm{GdCl}_{3}$ and ruthenium red increase the viability of astrocytes exposed to oxidative stress (Bai and Lipski, 2010). But for the lack of selectivity of these blockers, the role of TRPV4 in cerebral ischemia injury remains to be determined. HC-067047 is recently described as a potent and specific TRPV4 antagonist. It is reported that HC-067047 can inhibit mouse TRPV4 activation by various stimuli, including heat, hypotonic stimulation, AA, $4 \alpha-\mathrm{PDD}$, etc. (Everaerts et al., 2010). The selectivity of HC-067047 makes it more promising and possible to explore the involvement of TRPV4 in cerebral ischemia.

Hippocampus is highly susceptible to ischemia among brain regions and pyramidal neurons of CA1 region are most sensitive to ischemia damage (Schmidt-Kastner and Freund, 1991). Concerning that the entry of $\mathrm{Ca}^{2+}$ through NMDAR is the major pathway leading to excitotoxic cell death associated with ischemia, the present study firstly tested the effect of TRPV4 activation on NMDA-activated current $\left(I_{\mathrm{NMDA}}\right)$ in hippocampal CA1 pyramidal neurons and then explored the mechanisms underlying TRPV4-action. We also tested the effect of HC-067047 on brain infarction in focal cerebral ischemia model mice.

\section{MATERIALS AND METHODS ANIMALS}

Male mice (ICR, Oriental Bio Service Inc., Nanjing) were used in the study. Care of animals conformed to standards established by the National Institutes of Health. All animal protocols were approved by the Nanjing Medical University Animal Care and Use Committee (ID: 20110628). All efforts were made to minimize animal suffering and to reduce the number of animals used.

\section{SLICE PREPARATION}

Mice (3-week-old) were decapitated under deep anesthesia with ethyl ether. The brains were rapidly removed and the coronal brain slices $(400 \mu \mathrm{m})$ were cut using a vibrating microtome (Microslicer DTK 1500, Dousaka EM Co, Kyoto, Japan) in ice-cold modified artificial cerebrospinal fluid (mACSF) composed of (in $\mathrm{mM}$ ) $\mathrm{NaCl} 126, \mathrm{CaCl}_{2}$ 1, $\mathrm{KCl} 2.5, \mathrm{MgCl}_{2}$ 1, $\mathrm{NaHCO}_{3} 26, \mathrm{KH}_{2} \mathrm{PO}_{4} 1.25$, and D-glucose 20 oxygenated with a gas mixture of $95 \% \mathrm{O}_{2} / 5 \%$ $\mathrm{CO}_{2}$. After $1 \mathrm{~h}$ recovery, hippocampal slices were transferred to a recording chamber.

\section{ELECTROPHYSIOLOGICAL RECORDING}

Whole-cell patch clamp recording were performed at room temperature $\left(22-23^{\circ} \mathrm{C}\right)$. Hippocampal neurons were viewed with an upright microscope equipped with infrared-sensitive camera (DAGE-MTI, IR-1000). I NMDA was recorded using an EPC-10 amplifier (HEKA Elektronik, Lambrecht/Pfalz, Germany), sampled at $10 \mathrm{kHz}$ and filtered (Bessel) at $2.9 \mathrm{kHz}$. The capacitance and series resistance were compensated more than $90 \%$. Data obtained from neurons in which uncompensated series resistance resulted in voltage-clamp errors $>5 \mathrm{mV}$ were not taken in further analysis. Liquid junction potentials were compensated before patching. When the external solution was changed, measurements of the changes in liquid junction potentials were $<2 \mathrm{mV}$ and were not corrected.

The resistance of glass pipettes was 4-5 $\mathrm{M} \Omega$ filled with the pipette solution (in mM) CsCl 140, Tris-ATP 2, HEPES 10, EGTA 10 at $\mathrm{pH}$ 7.2. The slices were perfused continually with the oxygenated bath solution composed of (in $\mathrm{mM}$ ) $\mathrm{NaCl} 74, \mathrm{CaCl}_{2}$ 2.5, $\mathrm{KCl} 2.5, \mathrm{NaHCO}_{3} 26, \mathrm{KH}_{2} \mathrm{PO}_{4}$ 1.25, D-glucose 20, and Dmannitol 80 at osmolality of $300 \mathrm{mOsm} / \mathrm{kg}$. When recording $I_{\mathrm{NMDA}}$, hippocampal pyramidal neurons were held at $-60 \mathrm{mV}$. NMDA together with glycine $(10 \mu \mathrm{M})$ were dissolved in bath solution and focally applied using a rapid drug delivery system directed toward the soma of recorded neurons. One micrometer strychnine, $10 \mu \mathrm{M}$ bicuculline, $10 \mu \mathrm{M}$ NBQX, and $0.1 \mu \mathrm{M}$ tetrodotoxin (TTX) were added in bath solution to block glycine receptor, $\mathrm{GABA}_{\mathrm{A}}$ receptor, AMPA receptor, and voltage-gated sodium channels, respectively. When recording $4 \alpha$-PDD-evoked current, $10 \mu \mathrm{M} 4 \alpha$ PDD and $0.1 \mu \mathrm{M}$ TTX were added in $\mathrm{mASCF}$ and a ramp protocol depolarizing from -80 to $+80 \mathrm{mV}$ over $700 \mathrm{~ms}$ was used. Hypotonic solution was obtained by adjusting the concentration of $\mathrm{D}$ Mannitol. The osmolality was measured using the Advanced Micro Osmometer, model 3300 (Advanced instruments Inc., Norwood, MA, USA).

\section{DRUG TREATMENT}

For intracerebroventricular (icv) implantation, mice (weighing 25-30 g) were anesthetized with chloral hydrate. A guide cannula $(2.5 \mathrm{~mm}$ length, 23 gage) was implanted in the left lateral ventricle. HC-067047 stock solution was freshly diluted with $0.9 \%$ sodium chloride on the day of experiment. HC-067047 $(10 \mu \mathrm{mol} / 2 \mu \mathrm{l} / \mathrm{mouse})$ was injected with a stepper-motorized microsyringe (Stoelting, Wood Dale, IL, USA) at a rate of $0.5 \mathrm{ml} / \mathrm{min}$. Control mice were given an equal volume of vehicle. $\mathrm{HC}-067047$ was firstly injected $4 \mathrm{~h}(\mathrm{HC}-4 \mathrm{~h}), 8 \mathrm{~h}(\mathrm{HC}-8 \mathrm{~h})$, and $12 \mathrm{~h}$ (HC-12 h) after middle cerebral artery occlusion (MCAO), respectively, and then injected every $8 \mathrm{~h}$.

\section{PREPARATION OF FOCAL CEREBRAL ISCHEMIA MODEL}

Three days after cannula implantation, focal cerebral ischemia was induced by MCAO as previously described (Mulcahy et al., 2003). Briefly, after mice were anesthetized, a poly-L-lysine $(0.1 \%$, weight/volume)-coated nylon monofilament thread (3/0 gage with the tip heat blunted to a diameter of $0.104 \mathrm{~mm}$ ) was inserted through the external carotid artery and advanced into the internal carotid artery to occlude the origin of the middle cerebral artery (approximately $12 \mathrm{~mm}$ ). Adequacy of vascular occlusion and reperfusion was monitored in the front parietal cortex of the occluded side with a multichannel laser Doppler flow-meter (Perimed PF5050, Sweden). Body and head temperatures were controlled at $37 \pm 0.5^{\circ} \mathrm{C}$ with a thermostatically controlled heating pad. Arterial blood pressure and gases were monitored through a femoral catheter. After MCAO for $60 \mathrm{~min}$, the filament was withdrawn for reperfusion. Sham-operated (sham-op) animals were treated identically, except that the MCAs were not occluded after neck incision.

\section{INFARCTION VOLUME MEASUREMENT}

Brains were removed at $24 \mathrm{~h}$ post-MCAO, sectioned into five equidistant coronal slices (2-mm-thick), and incubated with a $2 \%$ 
2,3,5-triphenyl-tetrazolium chloride (TTC) solution for $20 \mathrm{~min}$ to visualize infarct tissue, using an image analysis software (NIHImage 3.12). Infarct volume was calculated as percentage of infarct area to the contralateral hemisphere area in each slice.

\section{CHEMICALS}

$4 \alpha$-Phorbol-12,13-didecanoate (4 $\alpha$-PDD) was obtained from Calbiochem (San Diego, CA, USA) and TTX was obtained from Enzo life Science (Ann Arbor, MI, USA). Others, unless stated, all came from Sigma Chemical Company.

4 $\alpha$-PDD, HC-067047, D-Sphingosine, bisindolylmaleimide II (BIM), TBB, DRB and KN62, NBQX, and strychnine were prepared as stock solutions in DMSO. The final concentration of DMSO in the bath chamber or pipette solution was $<0.1 \%$. KN93, $\mathrm{KN} 62$, and $\mathrm{D}$-Sphingosine were present in the pipette solution, while $\mathrm{D}(-)$-2-Amino-5-phosphonopentanoic acid (AP-5), ifenprodil, PEAQX tetrasodium hydrate (NVP-AAM007), 4 $\alpha$-PDD, HC-067047, BIM, phorbol-12-myristate 13-acetate (PMA), TBB, DRB, NBQX, strychnine, bicuculline, and strychnine were added in bath solution.

\section{DATA ANALYSIS}

Data are expressed as means \pm standard error and were analyzed with PulseFit (HEKA Elektronik) and Stata 7.0 software (STATA Corporation, USA). In the present study, after testing the effect of $4 \alpha$-PDD and hypotonicity on $I_{\mathrm{NMDA}}, 10 \mu \mathrm{M} 4 \alpha$-PDD was applied to the same neuron to test whether the neuron had TRPV4 receptors. All data came from the neurons where $4 \alpha$-PDD-evoked current could be recorded. Before data analysis, both normality of the distribution and homogeneity of variance were assessed and data transformation (logarithmic or square root transformation) was considered whenever necessary. Paired or unpaired $t$-test and ANOVA followed by Bonferroni's post hoc test were used for statistical analysis with the significance level set at $P<0.05$. In dose-response curve, $I_{\mathrm{NMDA}}$ induced by different dose of NMDA was normalized to $I_{\mathrm{NMDA}}$ induced by $300 \mu \mathrm{M}$ NMDA in isotonic solution $(300 \mathrm{mOsm} / \mathrm{kg})$ in the same neuron. The data were fitted to Logistic equation in which $I=I_{\max } /\left[1+\left(\mathrm{EC}_{50} / C\right)^{n}\right]$, with $n$ being Hill coefficient and $\mathrm{EC}_{50}$ being the concentration producing $50 \%$ maximal response. When exploring current-voltage relationship ( $I-V$ curve), $I_{\mathrm{NMDA}}$ induced at different holding potential was normalized to $I_{\mathrm{NMDA}}$ with the holding potential being $-60 \mathrm{mV}$ in isotonic solution in the same neuron.

\section{RESULTS \\ $4 \alpha$-PDD INCREASES INMDA IN HIPPOCAMPAL CA1 PYRAMIDAL NEURONS}

As glutamate excitotoxicity is of great importance in cerebral ischemia injury, we firstly explored whether TRPV4 activation modulated NMDAR function. After application of $30 \mu \mathrm{M}$ NMDA (for $20 \mathrm{~s}$ ) and its co-agonist glycine onto hippocampal CA1 pyramidal neuron an inward current was recorded. This $I_{\mathrm{NMDA}}$ was blocked by the specific NMDAR antagonist AP-5 (50 $\mu \mathrm{M})$, suggesting its mediation by NMDAR (Figure 1B). Here, it was found that $I_{\mathrm{NMDA}}$ was markedly increased by $31.6 \pm 2.1 \%$ from $-24.67 \pm 1.04$ to $-32.33 \pm 2.78 \mathrm{pA} / \mathrm{pF}$ after application of TRPV4 agonist $4 \alpha-$ PDD $(10 \mu \mathrm{M})$ for $5 \mathrm{~min}(n=10$, paired $t$-test, $P<0.01)$. The increase in $I_{\mathrm{NMDA}}$ was reversible after $4 \alpha$-PDD was washed out (Figure 1A). It was noted that in the presence of AP-5, $4 \alpha-\mathrm{PDD}$ almost had no effect on the current $(n=6$, paired $t$-test, $P>0.05$; Figure 1B).

We then studied the effect of $4 \alpha$-PDD on dose-response curve of $I_{\mathrm{NMDA}} \cdot \mathrm{EC}_{50}$ and $n$ values of dose-response curve were $19.91 \pm 1.74 \mu \mathrm{M}$ and 1.74 in the absence of $4 \alpha-\mathrm{PDD}$, respectively. After application of $4 \alpha-\mathrm{PDD}$, the maximal response to $300 \mu \mathrm{M}$ NMDA was markedly increased $(n=6$, paired $t$-test, $P<0.01)$, but $\mathrm{EC}_{50}(19.93 \pm 1.67 \mu \mathrm{M})$ and $n$ values $(1.63)$ were almost unaffected (unpaired $t$-test, $P>0.05$ in each case; Figure 1C).

We also performed experiments on current-voltage relationship of $I_{\mathrm{NMDA}}$. Application of $4 \alpha$-PDD markedly increased $I_{\mathrm{NMDA}}$ at different voltages ranging from -80 to $+60 \mathrm{mV}$. For example, when the holding potential was $-80 \mathrm{mV}, I_{\mathrm{NMDA}}$ was significantly increased from -27.90 to $-35.95 \mathrm{pA} / \mathrm{pF}(n=8$, paired $t$-test, $P<0.01)$. In $I-V$ curve of $I_{\mathrm{NMDA}}$, the reversal potential was $9.61 \pm 1.83 \mathrm{mV}$, which was not significantly different from the control $(9.29 \pm 1.58 \mathrm{mV} ; n=8$, paired $t$-test, $P>0.05)$. Besides this, we also compared the ratio of current at $+60 /-80 \mathrm{mV}$ to find that the ration was not affected by $4 \alpha-\mathrm{PDD}$ (control: $-0.28 ; 4 \alpha-\mathrm{PDD}$ : $-0.29, n=8$, paired $t$-test, $P>0.05$; Figure 1D).

\section{HYPOTONIC STIMULATION INCREASES I IMDA IN HIPPOCAMPAL CA1 PYRAMIDAL NEURONS}

As a cellular osmotic sensor, TRPV4 is sensitive to hypotonic stimuli. Here, we tested the effect of hypotonic stimulation on $I_{\mathrm{NMDA}}$. When the external solution was changed from isotonicity $(300 \mathrm{mOsm} / \mathrm{kg})$ to hypotonicity $(240 \mathrm{mOsm} / \mathrm{kg}), I_{\mathrm{NMDA}}$ was increased by $39.0 \pm 5.4 \%$ from $-25.01 \pm 2.71$ to $-34.13 \pm 2.82$ $\mathrm{pA} / \mathrm{pF}$ ( $n=17$, paired $t$-test, $P<0.05$; Figure $2 \mathrm{~A}$ ). The increase in $I_{\mathrm{NMDA}}$ by hypotonic stimulation was largely reversible after hypotonicity was washed out for $5 \mathrm{~min}$. The increase in $I_{\mathrm{NMDA}}$ was more evident with larger osmotic gradient (Figure 2E), and the following experiments were performed using hypotonic stimulation of $240 \mathrm{mOsm} / \mathrm{kg}$ which produced the significant increase in $I_{\mathrm{NMDA}}$.

As shown in Figure 2B, in the presence of AP-5, the current was almost unaffected by hypotonic stimulation $(n=6$, paired $t$-test, $P>0.05)$. In hypotonic solution, the maximal $I_{\mathrm{NMDA}}$ was significantly increased, but $\mathrm{EC}_{50}$ and $n$ values of dose-response curve were not markedly different in isotonic and hypotonic condition (paired $t$-test, $P>0.05$ in each case; Figure 2C). Hypotonic stimulation increased $I_{\mathrm{NMDA}}$ at every voltage ranging from -80 to $+60 \mathrm{mV}$, leaving the reversal potential (control: $9.52 \pm 1.31 \mathrm{mV} ; 240 \mathrm{mOsm} / \mathrm{kg}: 10.02 \pm 1.56 \mathrm{mV}, n=9$, paired $t$-test, $P>0.05)$ and $I(+60 \mathrm{mV}) / I(-80 \mathrm{mV})$ ration unaffected (control: $-0.28 ; 240 \mathrm{mOsm} / \mathrm{kg}:-0.29, n=9$, unpaired $t$-test, $P>0.05$; Figure 2D). These results indicate that both $4 \alpha-\mathrm{PDD}$ and hypotonic stimulation have similar effect on $I_{\mathrm{NMDA}}$.

\section{THE SPECIFIC TRPV4 ANTAGONIST HC-067047 ATTENUATES 4 $\alpha$-PDD- AND HYPOTONICITY-INDUCED INCREASE IN $I_{\text {NMDA }}$}

HC-067047 is a recently discovered specific TRPV4 antagonist (Everaerts et al., 2010). The present study showed that pre-application of HC-067047 $(1 \mu \mathrm{M})$ markedly attenuated the increase in $I_{\mathrm{NMDA}}$ by both hypotonicity and $4 \alpha$-PDD (unpaired 

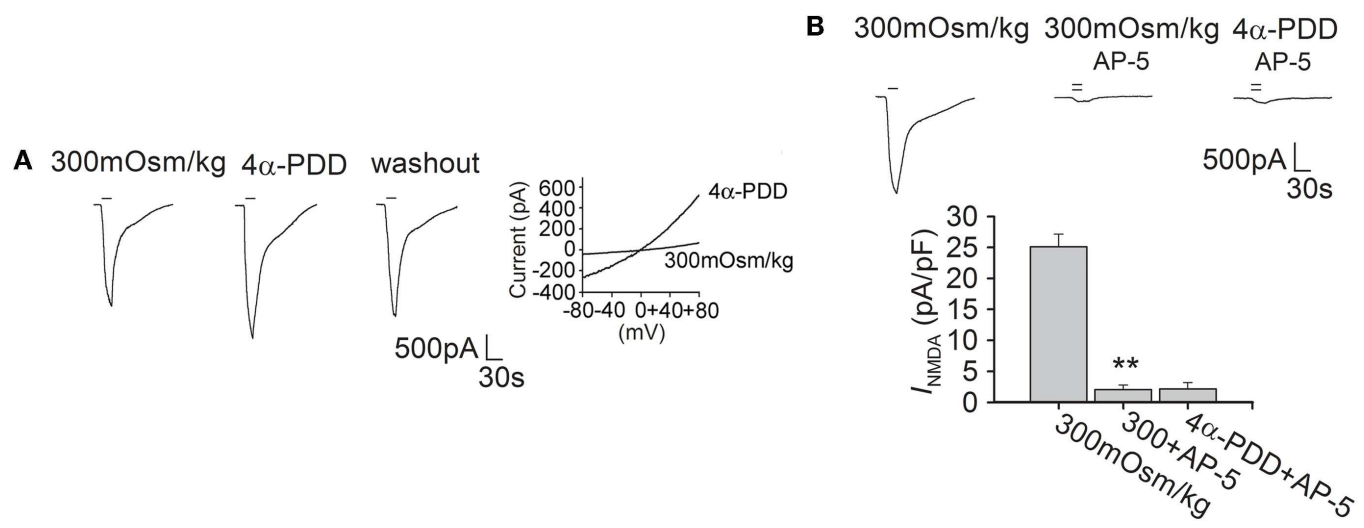

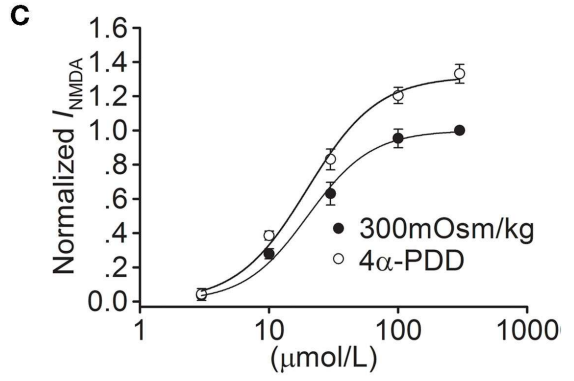

FIGURE 1 | $4 \alpha$-PDD increases $I_{\mathrm{NMDA}}$ in hippocampal CA1 pyramidal neurons. (A) The typical recordings show that $I_{\text {NMDA }}$ was increased from -1.93 to $-2.52 \mathrm{nA}$ after application of $4 \alpha$-PDD for $5 \mathrm{~min}$ and the current recovered to $-2.1 \mathrm{nA}$ after washout. $4 \alpha-P D D-e v o k e d$ current was recorded in the same neuron. (B) / IMDA was reduced from $-25.13 \pm 2.01$ to $-2.05 \pm 0.77$

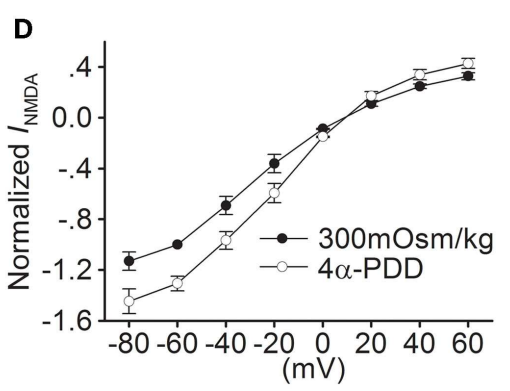

$t$-tests, $P<0.01$ in each case; Figure 3). Combined with the above results, it is suggested that activation of TRPV4 by either hypotonicity or $4 \alpha-\mathrm{PDD}$ enhances $I_{\mathrm{NMDA}}$. The following experiments were performed in isotonic and hypotonic solution to explore the possible mechanisms underlying TRPV4-mediated increase in $I_{\mathrm{NMDA}}$.

\section{NR2B SUBUNIT IS INVOLVED IN HYPOTONICITY-INCREASED $\boldsymbol{I}_{\text {NMDA }}$}

Functional NMDAR is composed of both an NR1 subunit, which contains the glycine binding site, and an NR2 (A-D) subunit, which binds to glutamate. In the adult brain, both NR2A and NR2B subunits are prominent in the hippocampus (Laurie et al., 1997). In the presence of ifenprodil $(10 \mu \mathrm{M})$, a specific NR2B subunit inhibitor, hypotonicity-induced increase in $I_{\mathrm{NMDA}}$ was markedly attenuated ( $n=33$, unpaired $t$-test, $P<0.01$; Figure $4 \mathrm{~A}$ ). By contrast, pre-application of NVP-AAM007 (0.3 $\mu \mathrm{M})$, a specific inhibitor of NR2A subunit, the increase in $I_{\mathrm{NMDA}}$ by hypotonicity was unaffected ( $n=29$, unpaired $t$-test, $P>0.05$; Figure 4B).

\section{CALCIUM/CALMODULIN-DEPENDENT PROTEIN KINASE II SIGNALING PATHWAYS IS INVOLVED IN HYPOTONICITY-INCREASED $I_{\text {NMDA }}$}

The NMDAR subunits possess phosphorylation sites for protein kinases that can modulate the function of NMDAR (Chen and Roche, 2007). The following experiments were performed to test whether Calcium/calmodulin-dependent protein kinase II (CaMKII), protein kinase C (PKC), and casein kinase II (CKII)
$\mathrm{pA} / \mathrm{pF}$ by AP-5 $(n=6$, paired $t$-test, $P<0.01)$. Note that in the presence of AP-5, the current was not changed by $4 \alpha-P D D .{ }^{*} P<0.01$ vs. $300 \mathrm{mOsm} / \mathrm{kg}$ (C) Dose-response curves for $I_{\mathrm{NMDA}}$ before and during $4 \alpha-\mathrm{PDD}$ application. Each point represents the normalized current from 6 to 10 neurons. (D) I-V curve was shown in the presence of and absence of $4 \alpha-P D D$. pathways were responsible for hypotonicity-increased $I_{\mathrm{NMDA}}$. As CaMKII plays an important role in phosphorylation of NMDAR, here we firstly evaluated the effect of CaMKII antagonists KN62 and KN93 on $I_{\text {NMDA }}$ in isotonic solution. Pre-incubation of KN62 $(5 \mu \mathrm{M})$ or KN93 $(5 \mu \mathrm{M})$ decreased $I_{\mathrm{NMDA}}$ from $25.50 \pm 1.15$ to $-21.01 \pm 2.71 \mathrm{pA} / \mathrm{pF}(n=7$, paired $t$-test, $P<0.05)$ and from $-25.08 \pm 2.14$ to $-20.06 \pm 1.56 \mathrm{pA} / \mathrm{pF}(n=8$, paired $t$ test, $P<0.05$ ), respectively. As shown in Figure 5A, with KN62 or KN93 in the pipette solution, $I_{\mathrm{NMDA}}$ was increased $8.5 \pm 3.8 \%$ $(n=15)$ and $8.7 \pm 3.6 \%(n=17)$ by hypotonicity, respectively, both of which were significantly different from hypotonicityincreased $I_{\mathrm{NMDA}}$ without antagonism of CaMKII (unpaired $t$ test, $P<0.01$ in each case). This result suggests that CaMKII is responsible for the increase in $I_{\mathrm{NMDA}}$ caused by TRPV4 activation.

In isotonic solution, $I_{\mathrm{NMDA}}$ was increased from $-24.42 \pm 2.78$ to $-27.51 \pm 0.84 \mathrm{pA} / \mathrm{pF}$ by PMA (agonist of PKC, $1 \mu \mathrm{M} ; n=6$, paired $t$-test, $P<0.05)$. After pre-application of $\mathrm{PKC}$ antagonists D-Sphingosine $(20 \mu \mathrm{M})$ or BIM $(1 \mu \mathrm{M}), I_{\mathrm{NMDA}}$ was decreased from $-24.69 \pm 0.94$ to $-21.63 \pm 1.33 \mathrm{pA} / \mathrm{pF}(n=9$, paired $t$-test, $P<0.05)$ and from $-25.04 \pm 1.55$ to $-22.63 \pm 2.64 \mathrm{pA} / \mathrm{pF}(n=7$, paired $t$-test, $P<0.05)$, respectively. Figure $5 \mathrm{~B}$ shows that preincubation of $\mathrm{D}$-Sphingosine or BIM did not affect the increase in $I_{\mathrm{NMDA}}$ by hypotonicity (unpaired $t$-test, $P>0.05$ in each case). We also tested the role of CKII signaling pathway, for this pathway is reported to specially phosphorylate NR2B subunit. Here, it was found that application of CKII antagonist TBB $(10 \mu \mathrm{M})$ or DRB 
B $300 \mathrm{mOsm} / \mathrm{kg} 300 \mathrm{mOsm} / \mathrm{kg} 240 \mathrm{mOsm} / \mathrm{kg}$

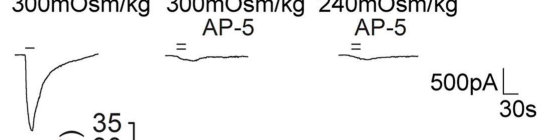

A $300 \mathrm{mOsm} / \mathrm{kg} 240 \mathrm{mOsm} / \mathrm{kg}$ washout
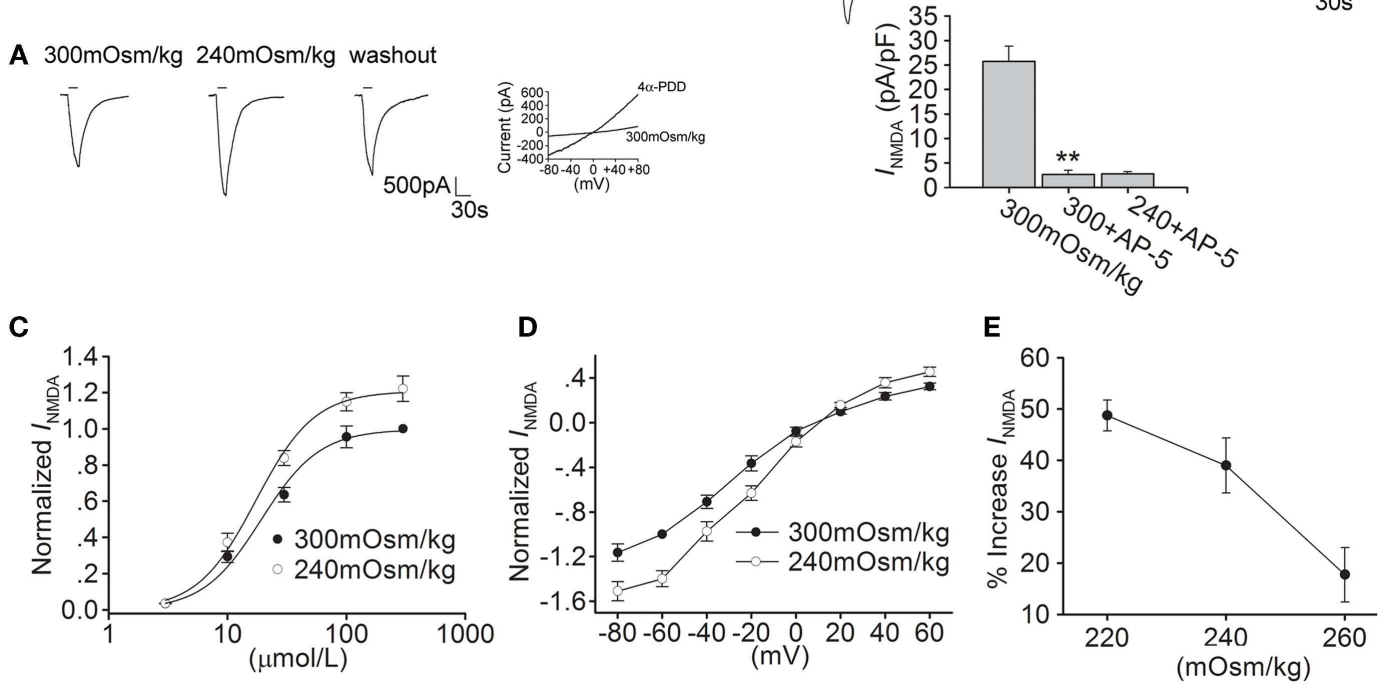

FIGURE 2 | Hypotonic stimulation increases $I_{\mathrm{NMDA}}$ in hippocampal CA1 pyramidal neurons. (A) The typical recordings show that $I_{\mathrm{NMDA}}$ was increased from -1.73 to $-2.42 \mathrm{nA}$ when the extracellular isotonic solution $(300 \mathrm{mOsm} / \mathrm{kg})$ was changed to hypotonic solution ( $240 \mathrm{mOsm} / \mathrm{kg}$ ) and the current recovered to $-1.81 \mathrm{nA}$ after washout. $4 \alpha$-PDD-evoked current was recorded in the same neuron. (B) / IMDA was reduced from $-25.74 \pm 3.12$ to $-2.67 \pm 0.87 \mathrm{pA} / \mathrm{pF}$ by AP-5 $(n=6$, paired $t$-test, $P<0.01$ ). Note that in the presence of AP-5, the current was not changed by hypotonic stimulation. ${ }^{*} P<0.01$ vs. $300 \mathrm{mOsm} / \mathrm{kg}$. (C) Dose-response curves for $I_{\mathrm{NMDA}}$ in isotonic and hypotonic solution. Each point represents the normalized current from 7 to 17 hippocampal neurons. $E_{50}$ values were $19.23 \pm 1.89$ and $18.24 \pm 1.07 \mu \mathrm{M}$, and $n$ were 1.71 and 1.79 for isotonicity and hypotonicity, respectively. (D) $1-V$ curves were shown in isotonic and hypotonic solution. (E) The plot shows that hypotonic stimuli exhibited

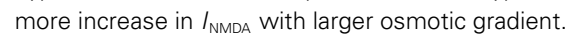

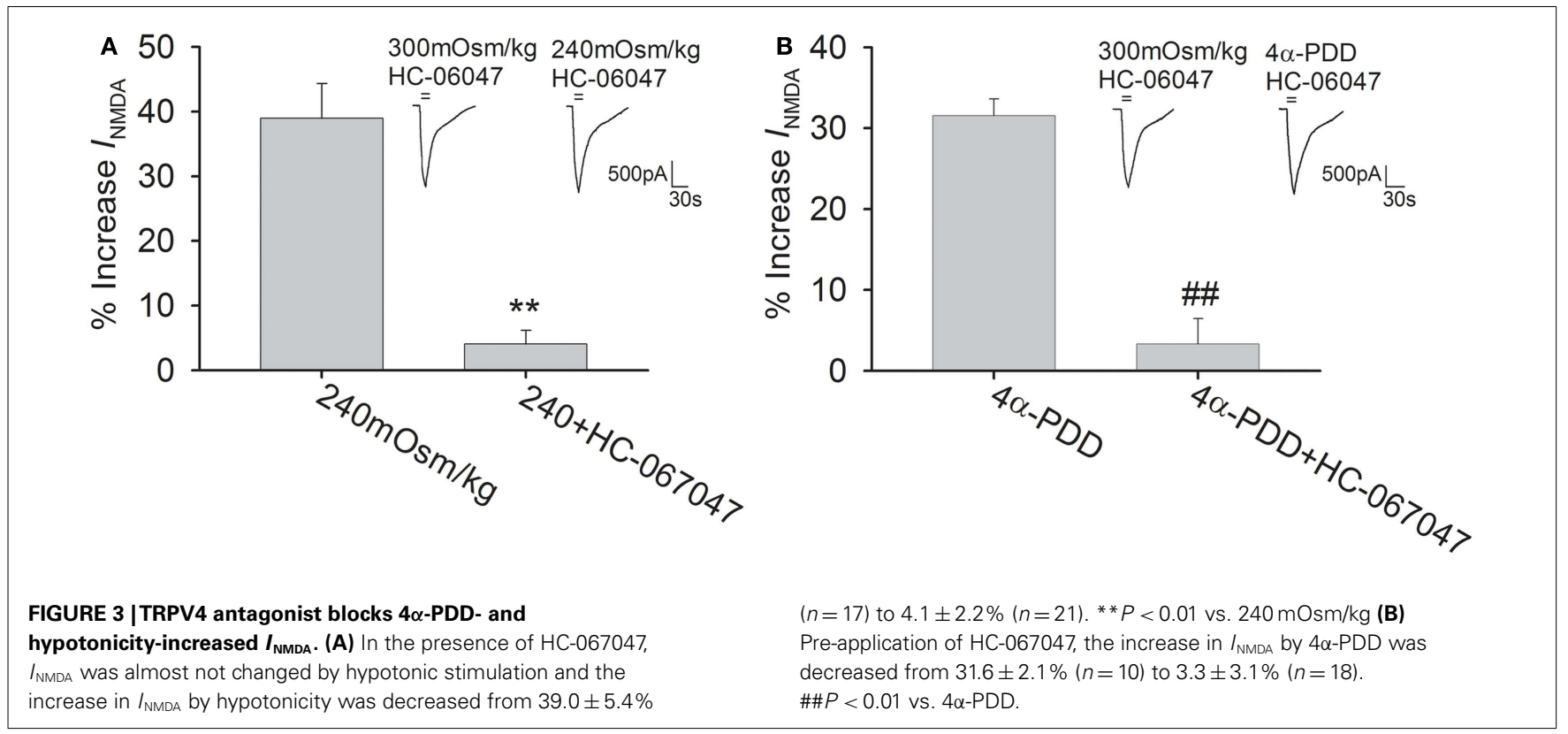

$(100 \mu \mathrm{M})$ decreased $I_{\mathrm{NMDA}}$ from $-25.01 \pm 5.95$ to $-18.19 \pm 2.50$ $\mathrm{pA} / \mathrm{pF}(n=7$, paired $t$-test, $P<0.01)$, and from $-24.94 \pm 1.49$ to $-17.16 \pm 1.57 \mathrm{pA} / \mathrm{pF}(n=7$, paired $t$-test, $P<0.01)$, respectively. Figure 5C shows that in the presence of $\mathrm{TBB}$ or DRB, $I_{\mathrm{NMDA}}$ was increased $41.1 \pm 4.0 \%(n=24)$ and $40.2 \pm 4.7 \%(n=10)$ by hypotonicity, respectively, both of which were similar to the increase in $I_{\mathrm{NMDA}}$ by hypotonicity alone (unpaired $t$-test, $P>0.05$ in each case). These results indicate that neither PKC nor 


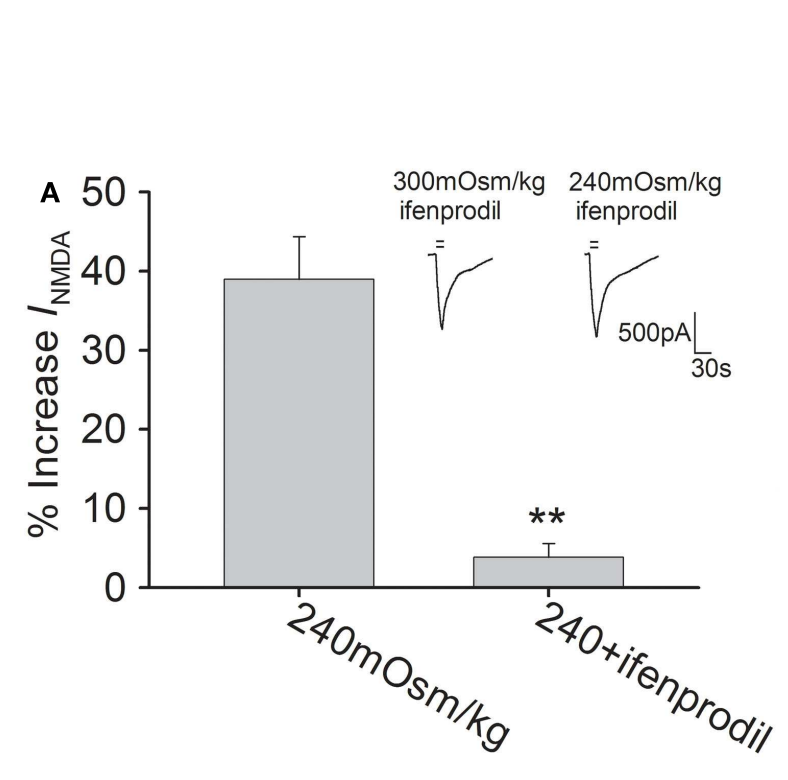

FIGURE 4 | NR2B subunit antagonist attenuates hypotonicity-increased $\boldsymbol{I}_{\mathrm{NMDA}}$. (A) In the presence of ifenprodil, the current was almost not changed by hypotonic stimulation and the increase in $I_{\text {NMDA }}$ by hypotonicity was markedly attenuated from

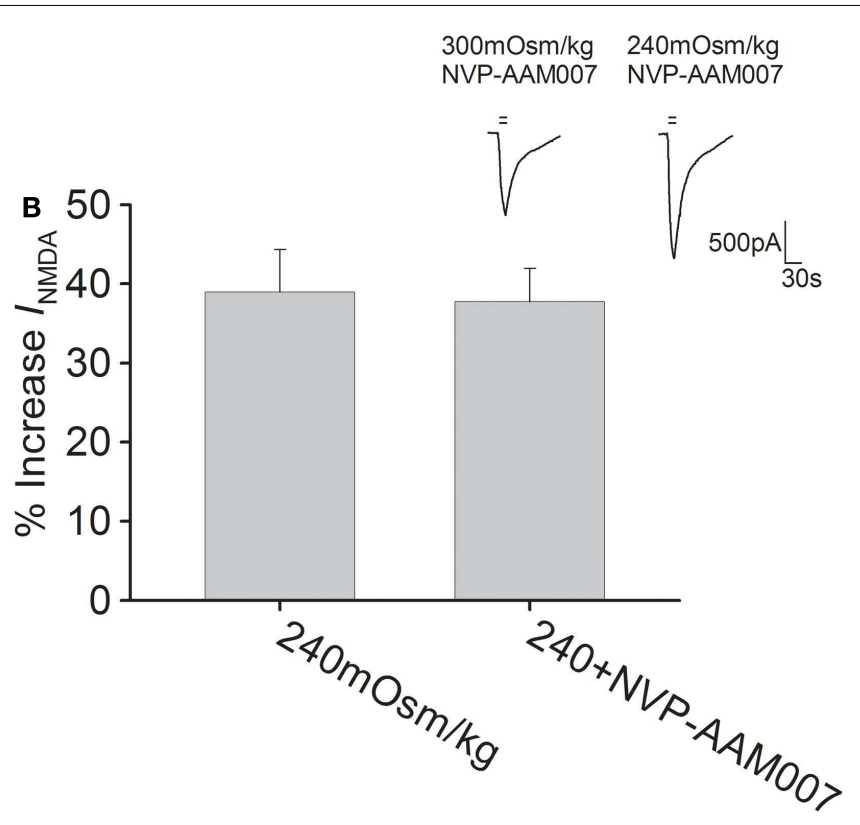

$39.0 \pm 5.4 \%(n=17)$ to $3.8 \pm 1.8 \%(n=18) .{ }^{*} P<0.01$ vs. $240 \mathrm{mOsm} / \mathrm{kg}$ (B) Pre-application of NVP-AAM007, I IMDA was increased $37.8 \pm 4.2 \%(n=14)$ by hypotonic stimulation, which was not different from the increase by hypotonicity alone.
CKII signaling system is involved in TRPV4 activation-induced increased $I_{\mathrm{NMDA}}$.

\section{TRPV4 ANTAGONIST REDUCES BRAIN DAMAGE AFTER FOCAL CEREBRAL ISCHEMIA}

The neuroprotection of blocking TRPV4 was tested in vivo using MCAO mice to induce focal cerebral ischemia. Figure 6A shows a representative experiment that the area of non-viable tissue, as indicated by pale color, was much smaller $(3.0 \pm 1.8 \%, n=10)$ in the infracted hemisphere when mice were treated with HC067047 (HC-4h), compared with the mice treated with vehicle (35.3 $\pm 4.2 \%, n=10$; ANOVA followed by Bonferroni's post hoc test, $P<0.01)$. Furthermore, HC-067047, when was firstly administered $8 \mathrm{~h}(\mathrm{HC}-8 \mathrm{~h}, 17.1 \pm 5.8 \%, n=10)$ or $12 \mathrm{~h}(\mathrm{HC}-12 \mathrm{~h}$, $18.2 \pm 4.1 \%, n=10$ ) post-MCAO, could markedly reduce the size of infarction (ANOVA followed by Bonferroni's post hoc test, $P<0.05$ in each case; Figure 6B). These results suggest that blockage of TRPV4 reduces MCAO-induced cerebral injury with an efficacious time-window of at least $12 \mathrm{~h}$.

\section{DISCUSSION}

Cytotoxic edema is an important pathological process as well as a major cause of neuronal death in cerebral ischemia injury (Marmarou, 2007; Simard et al., 2007). Activation of TRPV4 is likely for its sensitivity to cell swelling, AA, and its metabolism EETs, which are always associated with cerebral ischemia. During ischemia, glutamate-induced intracellular calcium overload, which is mediated through NMDAR, plays an important role in neuronal injury. In this study, we found that application of
$4 \alpha$-PDD or hypotonic stimulation enhanced $I_{\mathrm{NMDA}}$ in hippocampal CA1 pyramidal neurons, which was markedly attenuated by the specific TRPV4 antagonist HC-067047 (Figure 3), indicating that activation of TRPV4 leads to the increase in $I_{\mathrm{NMDA}}$. Additionally, $4 \alpha$-PDD- or hypotonicity-increased $I_{\mathrm{NMDA}}$ was blocked by NMDAR antagonist AP-5, indicating that TRPV4 potentiates NMDAR response (Figures $\mathbf{1 B}$ and $\mathbf{2 B}$ ). It is known that NMDARs are calcium-permeable and the opening of these channels leads to further membrane depolarization and greater calcium influx, exacerbating intracellular calcium overload. Therefore, TRPV4induced enhancement of NMDAR response may helps to facilitate glutamate-neurotoxicity during stroke.

The increase in $I_{\mathrm{NMDA}}$ probably results from an increase in NMDAR expression or enhanced NMDAR function. The present study mainly focused on the latter, for there is a report that NMDAR subunits expression is unaffected in TRPV4 knockout mice (Shibasaki et al., 2007). Although the maximal $I_{\mathrm{NMDA}}$ was increased, $\mathrm{EC}_{50}$ value in dose-response curve was not changed by either $4 \alpha$-PDD or hypotonicity (Figures 1C and 2C), indicating that TRPV4-action is not dependent on increasing ligand binding affinity. Additionally, $4 \alpha-\mathrm{PDD}$ and hypotonicity did not change the reversal voltage or $I(+60 \mathrm{mV}) / I(-80 \mathrm{mV})$ ration in $I-V$ curve (Figures 1D and 2D), indicating that TRPV4-action is voltage-independent. It has been demonstrated that NR2B subunit is critical for a number of basic structural and functional attributes associated with NMDAR (Loftis and Janowsky, 2003). In this study, selective antagonism of NR2B but not NR2A subunit markedly blocked hypotonicity-induced increase in $I_{\mathrm{NMDA}}$ (Figure 4), suggesting the involvement of NR2B subunit in TRPV4-increased $I_{\text {NMDA }}$. Increasing evidence supports the notion that the level of 

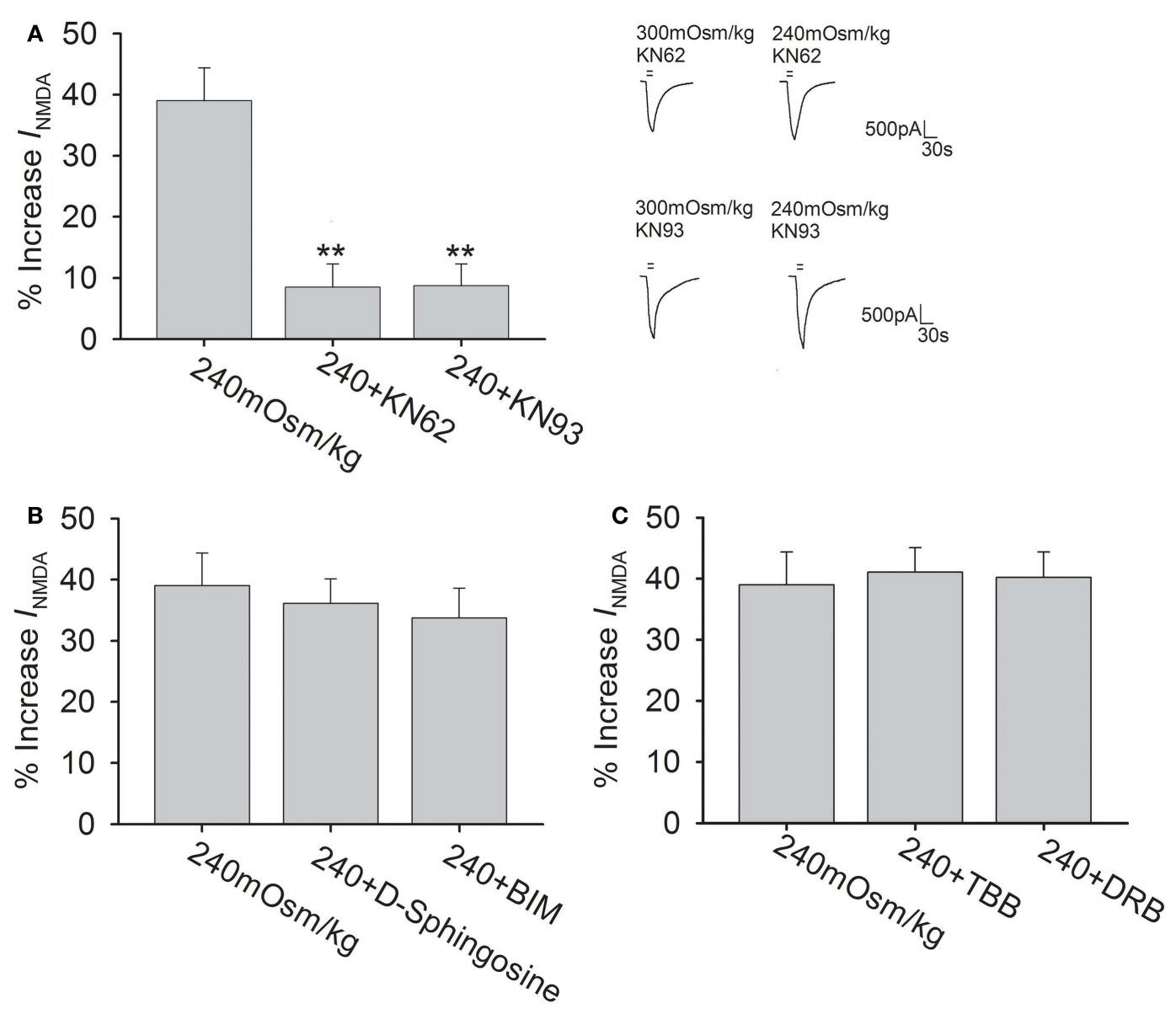

FIGURE 5 | CaMKII antagonists attenuate hypotonicity-increased $I_{\text {NMDA. }}$ (A) In the presence of KN62 or KN93, I IMDA was almost not affected by hypotonic stimulation and the increase in / NMDA by hypotonicity was markedly blocked by pre-application of KN62 or KN93. ${ }^{*} P<0.01 \mathrm{vs.} 240 \mathrm{mOsm} / \mathrm{kg}$. (B)
$I_{\text {NMDA }}$ was increased $36.2 \pm 4.0 \%(n=20)$ and $33.7 \pm 4.9 \%(n=12)$ by hypotonic stimulation in the presence of $d$-sphingosine or BIM, respectively. (C) Pre-incubation of TBB or DRB did not affect the increase in IMDA $_{\text {by }}$ hypotonicity.

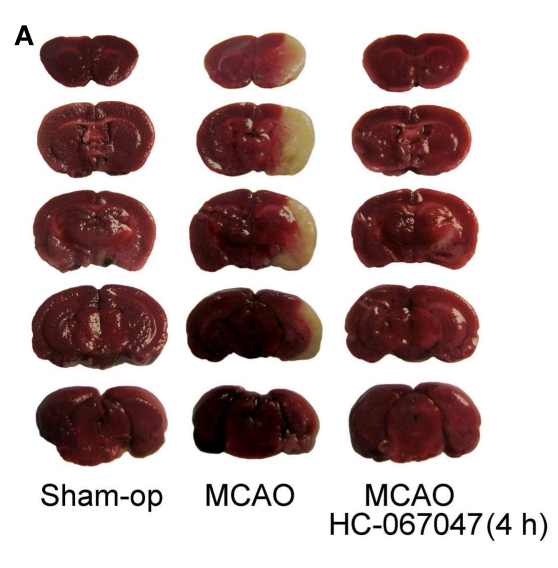

FIGURE 6 |TRPV4 antagonist reduces brain damage after focal cerebral ischemia. (A) Representative photographs of cerebral infarction in MCAO mice treated with $\mathrm{HC}-067047$. Note that treatment with $\mathrm{HC}-067047$ (HC-4 h) after stroke significantly reduced the MCAO-induced cerebral infarction. (B)

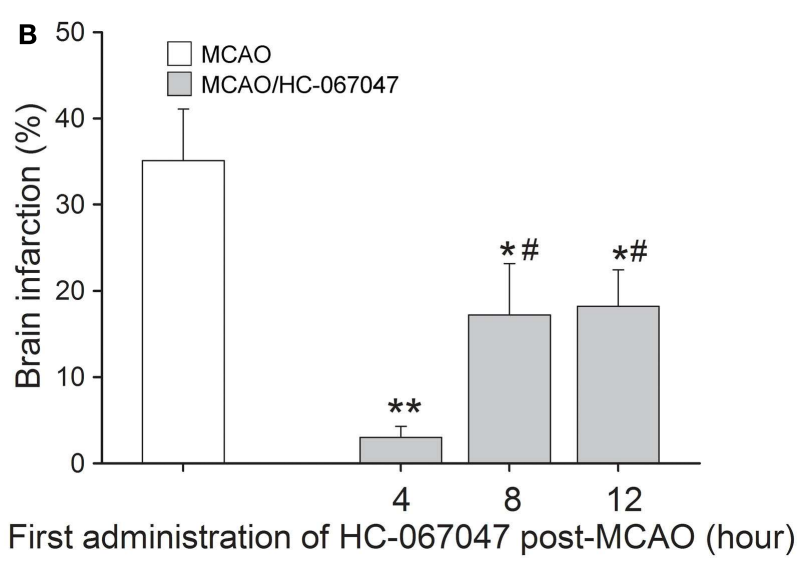

Time-window of neuroprotective effect of HC-067047. Bar graphs show the mean infarct volume percentage in MCAO mice that were treated with HC-067047 starting 4, 8, and $12 \mathrm{~h}$ post-MCAO. ${ }^{*} P<0.01$ and ${ }^{*} P<0.05$ vs. vehicle-treated MCAO mice, $\# P<0.05$ vs. HC-4 h. 
NR2B subunit phosphorylation is linked to the receptor activity, leading to the prolonged calcium influx. Our previous studies have reported that some intracellular signaling pathways are responsible for hypotonicity (TRPV4)-induced modulation on voltagegated ion channels and TRPV1 receptor (Liu et al., 2007; Chen et al., 2008a,b, 2009; Li et al., 2011b). Here, CaMKII, PKC, and CKII signaling pathways were selected for they are responsible for phosphorylation of NR2B subunit (Chen and Roche, 2007). We found that the increase in $I_{\mathrm{NMDA}}$ by hypotonic stimulation was markedly attenuated by CaMKII, but not PKC or CKII antagonists (Figure 5). These results indicate that the phosphorylation of NR2B subunit by CaMKII is responsible for TRPV4-induced increase in $I_{\mathrm{NMDA}}$. It is known that activation of CaMKII is necessary before it phosphorylates NMDAR, which is dependence on $\mathrm{Ca}^{2+} / \mathrm{CaM}$. TRPV4 activation increased $I_{\mathrm{NMDA}}$, which may facilitate calcium influx through NMDAR and exacerbate the increase of $\left[\mathrm{Ca}^{2+}\right]_{\mathrm{i}} \cdot \mathrm{Mg}^{2+}$, which mainly exists inside of cell in normal condition, is very important for the activation of some kinases. The present recording solution was lack of $\mathrm{Mg}^{2+}$, suggesting that this recording condition may not be optimal condition for CaMKII activity. However, activation of CaMKII was still possible, which was caused by $\mathrm{Mg}^{2+}$ inside of neurons. Here, pre-application of CaMKII antagonists KN62 and KN93 inhibited $I_{\text {NMDA }}$ in isotonic solution, which was consistent with the above deduction. It is reported that $\mathrm{Ca}^{2+} /$ calmodulin-activated Ser-Thr kinase (CASK), which functions as an active protein kinase even without $\mathrm{Mg}^{2+}$ binding, may associated with CaMKII and modulate its phosphorylation state (Mukherjee et al., 2008). Whether CASK is involved CaMKII-induced modulation of NMDAR function remains unclear in the present study. On the other hand, the $\mathrm{C}$ terminus of TRPV4 contains a PDZ-binding-like motif that may contribute to the interaction of TRPV4 with PDZ-domain proteins (Garcia-Elias et al., 2008). Therefore, there may be other possibility that TRPV4 per se influences the activation of CaMKII.

Another important result of this study is that we firstly provide in vivo evidence that closing TRPV4 exerts potent neuroprotection against cerebral ischemic injury with at least a $12 \mathrm{~h}$ efficacious time-window (Figure 6). In fact, over-activation of TRPV4 may induce neuronal injury in cerebral ischemia through multiple mechanisms. In cultured hippocampal pyramidal neurons, application of glutamate induces more $\left[\mathrm{Ca}^{2+}\right]_{i}$ increase at $37^{\circ} \mathrm{C}$ than at $25^{\circ} \mathrm{C}$, which is more evident in wild type neurons than in TRPV4 knockout neurons. Moreover, this temperaturedependent $\left[\mathrm{Ca}^{2+}\right]_{\mathrm{i}}$ increase at $37^{\circ} \mathrm{C}$ was markedly inhibited by a NMDAR blocker in wild type but not in TRPV4 knockout neurons, which indicates that TRPV4 activation promotes NMDAR activation in hippocampal pyramidal neurons (Shibasaki et al., 2007). Consistently, the present study showed that activation of TRPV4 enhanced $I_{\mathrm{NMDA}}$ in hippocampal CA1 pyramidal neurons. On the other hand, activation of TRPV4 can depolarize the resting membrane potential (Shibasaki et al., 2007), which helps the release of presynaptic glutamate. Our experiment performed on the excitatory postsynaptic current (EPSC) also showed that TRPV4 agonist $4 \alpha$-PDD increased EPSC in hippocampal slices (Figure A1 in Appendix), indicating that TRPV4 activation enhances synaptic transmission. Therefore, the enhancement of
NMDAR response or/and increase in glutamate release is likely involved in TRPV4-mediated neuronal injury during stroke.

TRPV4 forms calcium-permeable, non-selective cation channels (Plant and Strotmann, 2007). Many studies including ours have reported that activation of TRPV4R causes an increase in intracellular calcium (Liu et al., 2007; Plant and Strotmann, 2007). Reactive oxygen species (ROS) and nitric oxide (NO) are important pathophysiological mediators of ischemia-induced toxicity (Loh et al., 2006). Recent studies performed in the urothelial cells, human coronary arterial endothelial cells, and lung macrophages have reported that activation of TRPV4 can stimulate the production of $\mathrm{H}_{2} \mathrm{O}_{2}$ and $\mathrm{NO}$, which is mediated by TRPV4-induced increase in intracellular calcium (Donkó et al., 2010; Li et al., 2011a; Bubolz et al., 2012). Therefore, it is possible that during stroke, TRPV4 over-activation exacerbates ROS and NO production to induce neuronal injury.

It has recently been reported that TRPV4 and aquaporin-4 (AQP4) are co-expressed in astrocytic plasma membranes in situ, as well as in primary cultures and transfected cell lines (Benfenati et al., 2011). AQP4 plays an important role in keeping water balance in BBB and is involved in the formation of vasogenic brain edema (Zador et al., 2009). AQP4 and TRPV4 form a complex in the astrocytes that is essential for the brain's volume homeostasis by acting as an osmosensor (Benfenati et al., 2011). Moreover, TRPV4 may participate in the pathogenic mechanisms of astroglial reactivity following ischemic insult because it is involved in ischemia-induced calcium entry in reactive astrocytes (Butenko et al., 2012). TRPV4 antagonists increase the viability of astrocytes in oxidative stress-induced cell damage (Bai and Lipski, 2010). The experiment performed on primary cultures of human respiratory epithelial cells shows that TRPV4 mediates calcium influx into human bronchial epithelia upon exposure to diesel exhaust particle, which leads to the activation of matrix metalloproteinase-1 (MMP-1; Li et al., 2011a). MMP-2 and MMP-9 are able to digest the endothelial basal lamina, resulting in opening of BBB. After cerebral ischemia, levels of MMP-2 and MMP-9 are increased, which plays an active role in the formation of brain edema and the secondary brain injury. More experiments will be needed to reveal a possible involvement of TRPV4 activation and MMPs activation in ischemia brain. Therefore, TRPV4 over-activation may also be responsible for the formation of vasogenic brain edema through facilitating AQP4 function or exacerbating the injury of astrocytes or/and basement membrane to increase the permeability of BBB.

In conclusion, this study shows that activation of TRPV4 potentiates NMDAR response, which may facilitate and prolong the glutamate excitotoxicity. Therefore, closing TRPV4 may effectively inhibit $\left[\mathrm{Ca}^{2+}\right]_{\mathrm{i}}$ overload and avoid the side effects through not directly inhibiting NMDAR. Ischemic injury is a complex insult, and treatment with a cocktail for multi-target is a more effective therapeutic strategy. The neuroprotection of TRPV4 antagonist exhibits long time-window (at least $12 \mathrm{~h}$ ), which also indicates that the neuroprotective effect of closing TRPV4 may be mediated through multiple mechanisms. The present study suggests that TRPV4 is a promising novel target for treatment of ischemic stroke. 


\section{ACKNOWLEDGMENTS}

This work was supported by National Natural Science Foundation of China (31271206 and 30900577), Science and Technology Project of Jiangsu Province (BK2009416 and

\section{REFERENCES}

Bai, J. Z., and Lipski, J. (2010). Differential expression of TRPM2 and TRPV4 channels and their potential role in oxidative stress-induced cell death in organotypic hippocampal culture. Neurotoxicology 31, 204-214.

Benfenati, V., Caprini, M., Dovizio, M., Mylonakou, M. N., Ferroni, S., Ottersen, O. P., et al. (2011). An aquaporin-4/transient receptor potential vanilloid 4 (AQP4/TRPV4) complex is essential for cell-volume control in astrocytes. Proc. Natl. Acad. Sci. U.S.A. 108, 2563-2568.

Bubolz, A. H., Mendoza, S. A., Zheng, X., Zinkevich, N. S., Li, R., Gutterman, D. D., et al. (2012). Activation of endothelial TRPV4 channels mediates flow-induced dilation in human coronary arterioles: role of $\mathrm{Ca}^{2+}$ entry and mitochondrial ROS signaling. Am. J. Physiol. Heart Circ. Physiol. 302, H634-H642.

Butenko, O., Dzamba, D., Benesova, J., Honsa, P., Benfenati, V., Rusnakova, V., et al. (2012). The increased activity of TRPV4 channel in the astrocytes of the adult rat hippocampus after cerebral hypoxia/ ischemia. PLoS ONE 7:e39959. doi:10.1371/journal.pone.0039959

Chen, B. S., and Roche, K. W. (2007). Regulation of NMDA receptors by phosphorylation. Neuropharmacology 53, 362-368.

Chen, L., Liu, C., and Liu, L. (2008a). Changes in osmolality modulate voltage-gated calcium channels in trigeminal ganglion neurons. Brain Res. 1208, 56-66.

Chen, L., Liu, C., and Liu, L. (2008b). The modulation of voltage-gated potassium channels by an isotonicity in trigeminal ganglion neurons. Neuroscience 154, 482-495.

Chen, L., Liu, C., Liu, L., and Cao, X. (2009). Changes in osmolality modulate voltage-gated sodium channels in trigeminal ganglion neurons. Neurosci. Res. 64, 199-207.
Donkó, A., Ruisanchez, E., Orient, A., Enyedi, B., Kapui, R., Péterfi, Z., et al. (2010). Urothelial cells produce hydrogen peroxide through the activation of Duoxl. Free Radic. Biol. Med. 49, 2040-2048.

Everaerts, W., Zhen, X., Ghosh, D., Vriens, J., Gevaert, T., Gilbert, J. P., et al. (2010). Inhibition of the cation channel TRPV4 improves bladder function in mice and rats with cyclophosphamide-induced cystitis. Proc. Natl. Acad. Sci. U.S.A. 107, 19084-19089. R., and Valverde, M. A. (2008). IP3 receptor binds to and sensitizes TRPV4 channel to osmotic stimuli via a calmodulin-binding site. J. Biol. Chem. 283, 31284-31288.

Kauer, J. A., and Gibson, H. E. (2009). Hot flash: TRPV channels in the brain. Trends Neurosci. 32, 215-224.

Kemp, J. A., and McKernan, R. M. (2002). NMDA receptor pathways as drug targets. Nat. Neurosci. 5, 1039-1042.

Laurie, D. J., Bartke, I., Schoepfer, R., Naujoks, K., and Seeburg, P. H. (1997). Regional, developmental and interspecies expression of the four NMDAR2 subunits, examined using monoclonal antibodies. Brain Res. Mol. Brain Res. 51, 23-32.

Li, J., Kanju, P., Patterson, M., Chew, W. L., Cho, S. H., Gilmour, I., et al. (2011a). TRPV4-mediated calcium influx into human bronchial epithelia upon exposure to diesel exhaust particles. Environ. Health Perspect. 119, 784-793.

Li, L., Liu, C., Chen, L., and Chen, L. (2011b). Hypotonicity modulates tetrodotoxin-sensitive sodium current in trigeminal ganglion neurons. Mol. Pain 7, 27-32.

Lipski, J., Park, T. I., Li, D., Lee, S. C., Trevarton, A. J., Chung, K. K., et al. (2006). Involvement of TRPlike channels in the acute ischemic response of hippocampal CA1 neurons in brain slices. Brain Res. 1077, 187-199.
Garcia-Elias, A., Lorenzo, I. M., Vicente,

BK2011029), Basic Medical Advantage Disciplines Project of Nanjing Medical University (JX10131801055), and Graduate Students Scientific Research Innovation Project of Jiangsu Province (CXZZ12_0567).

Liu, L., Chen, L., Liedtke, W., and Simon, S. A. (2007). Changes in osmolality sensitize the response to capsaicin in trigeminal sensory neurons. J. Neurophysiol. 97, 2001-2015.

Loftis, J. M., and Janowsky, A. (2003). The N-methyl-D-aspartate receptor subunit NR2B: localization, functional properties, regulation, and clinical implications. Pharmacol. Ther. 97, 55-85.

Loh, K. P., Huang, S. H., De Silva, R., Tan, B. K., and Zhu, Y. Z. (2006). Oxidative stress: apoptosis in neuronal injury. Curr. Alzheimer Res. 3, 327-337.

Marmarou, A. (2007). A review of progress in understanding the pathophysiology and treatment of brain edema. Neurosurg. Focus 22, E1-E10.

Mukherjee, K., Sharma, M., Urlaub, H., Bourenkov, G. P., Jahn, R., Südhof, T. C., et al. (2008). CASK Functions as a $\mathrm{Mg}^{2+}$-independent neurexin kinase. Cell 133, 328-339.

Mulcahy, N. J., Ross, J., Rothwell, N. J., and Loddick, S. A. (2003). Delayed administration of interleukin-1 receptor antagonist protects against transient cerebral ischemia in the rat. Br. J. Pharmacol. 140, 471-476.

Paschen, W. (1996). Glutamate excitotoxicity in transient global cerebral ischemia. Acta Neurobiol. Exp. (Wars) 56, 313-322.

Plant, T. D., and Strotmann, R. (2007). TRPV4. Handb. Exp. Pharmacol. 179, 189-205.

Schmidt-Kastner, R., and Freund, T. F. (1991). Selective vulnerability of the hippocampus in brain ischemia. Neuroscience 40, 599-636.

Shibasaki, K., Suzuki, M., Mizuno, A. and Tominaga, M. (2007). Effects of body temperature on neural activity in the hippocampus: regulation of resting membrane potentials by transient receptor potential vanilloid 4. J. Neurosci. 27, 1566-1575.

Simard, J. M., Kent, T. A., Chen, M., Tarasov, K. V., and Gerzanich,
V. (2007). Brain oedema in focal ischaemia: molecular pathophysiology and theoretical implications. Lancet Neurol. 6, 258-268.

Sun, H. S., Jackson, M. F., Martin, L. J., Jansen, K., Teves, L., Cui, H., et al. (2009). Suppression of hippocampal TRPM7 protein prevents delayed neuronal death in brain ischemia. Nat. Neurosci. 12, 1300-1307.

Szydlowska, K., and Tymianski, M. (2010). Calcium, ischemia and excitotoxicity. Cell Calcium 47, 122-129. Westerberg, E., Deshpande, J. K., and Wieloch, T. (1987). Regional differences in arachidonic acid release in rat hippocampal CA1 and CA3 regions during cerebral ischemia. $J$. Cereb. Blood Flow Metab. 7, 189-192.

Zador, Z., Stiver, S., Wang, V., and Manley, G. T. (2009). Role of aquaporin-4 in cerebral edema and stroke. Handb. Exp. Pharmacol. 190, 159-170.

Conflict of Interest Statement: The authors declare that the research was conducted in the absence of any commercial or financial relationships that could be construed as a potential conflict of interest.

Received: 30 October 2012; accepted: 09 February 2013; published online: 04 March 2013.

Citation: $L i L, Q u$ W, Zhou L, Lu $Z$, Jie P, Chen L and Chen L (2013) Activation of transient receptor potential vanilloid 4 increases NMDA-activated current in hippocampal pyramidal neurons. Front. Cell. Neurosci. 7:17. doi: 10.3389/fncel.2013.00017

Copyright (C) $2013 \mathrm{Li}, \mathrm{Qu}, \mathrm{Zhou}, \mathrm{Lu}$, Jie, Chen and Chen. This is an openaccess article distributed under the terms of the Creative Commons Attribution License, which permits use, distribution and reproduction in other forums, provided the original authors and source are credited and subject to any copyright notices concerning any third-party graphics etc. 


\section{APPENDIX}

$300 \mathrm{mOsm} / \mathrm{kg} \quad 4 \alpha-P D D \quad$ washout

FIGURE A1 | Effect of $4 \alpha-$ PDD on EPSC in hippocampal slices. EPSCs recorded from $\mathrm{CA} 1$ pyramidal neurons of hippocampal slices before, during and after $4 \alpha-P D D(10 \mu \mathrm{M})$ application. On the average, EPSC slope was enhanced $42.1 \pm 5.0 \%$ by $4 \alpha-$ PDD $(n=8$, paired $t$-test, $P<0.01)$. EPSC was evoked by stimulating Schaffer fibers through a constant-current pulse delivered by a Master-8 Stimulator (AMPI, Jerusalem, Israel). Hippocampal CA1 neurons were held at $-70 \mathrm{mV}$ and perfused with oxygenated bath solution composed of (in $\mathrm{mM}$ ) $\mathrm{NaCl} 126, \mathrm{CaCl}_{2} 1, \mathrm{KCl} 2.5, \mathrm{MgCl}_{2} 1$,

$\mathrm{NaHCO}_{3} 26, \mathrm{KH}_{2} \mathrm{PO}_{4}$ 1.25, d-glucose 20, bicuculline 0.01, and TTX $0.1 \mu \mathrm{M}$.

Pipette solution was composed of (in $\mathrm{mM}$ ) Cs-gluconate $132.5, \mathrm{CsCl} 17.5$,

$\mathrm{MgCl}_{2}$ 2, EGTA 0.5, HEPES 10, ATP 4, and QX-314

[N-(2,6-Dimethylphenylcarbamoylmethyl) triethylammonium bromide] 5 and $\mathrm{pH}$ was adjusted to 7.2 by $\mathrm{CsOH}$. 